\title{
Finite-size scaling of correlation functions in one-dimensional Anderson-Hubbard model
}

\author{
Satoshi Nishimoto \\ Leibniz-Institut für Festkörper- und Werkstoffforschung Dresden, D-01171 Dresden, Germany \\ Tomonori Shirakawa \\ Department of Physics, Chiba University, Chiba 263-8522, Japan \\ Institut für Theoretische Physik, Leibniz Universität Hannover, D-30167 Hannover, Germany
}

(Dated: March 24, 2022)

\begin{abstract}
We study the one-dimensional Anderson-Hubbard model using the density-matrix renormalization group method. The influence of disorder on the Tomonaga-Luttinger-liquid behavior is quantitatively discussed. Based on the finite-size scaling analysis of density-density correlation functions, we find the following results: i) the charge exponent is significantly reduced by disorder at low filling and near half filling, ii) the localization length decays as $\xi \sim \Delta^{-2}$, where $\Delta$ is the disorder strength, independently of the on-site Coulomb interaction as well as band filling, and iii) the localization length is strongly suppressed by the on-site Coulomb interaction near half filling in association with the formation of the Mott plateaus.
\end{abstract}

PACS numbers: 71.10.Fd, 71.10.Pm, 71.23.-k, 71.15.Dx

We know that lattice disorder or defects in the electron system lead to various intriguing effects. One of the most famous examples is Anderson localization [1]; a large number of phenomena is explained in terms of the localization of quantum states [2]. Meanwhile, it is widely recognized that a full understanding of the experimentally observed phenomena has a need for taking correlation effects, as well as disorder, into account. Hence, the Anderson localization in the presence of electron-electron interactions has been an inevitable problem in the field of condensed matter physics [3|. A hot topic is the appearance of "zero bias anomaly [4]", i.e., a suppression of the density of states $A(E)$ at the Fermi energy $E_{\mathrm{F}}$, by the interplay between disorder and short-range Coulomb interaction [5, 6]. Recently, it was reported that the similar anomaly occurs even in one-dimensional (1D) system [7]. It is possibly related to a vanishing of the photoemission spectral weight around $E_{\mathrm{F}}$ (or "pseudogap-like" behavior) observed in quasi-1D charge-transfer materials TTFTCNQ and Bechgaad salts (TMTSF) ${ }_{2} \mathrm{X}[8,9]$.

Generally, 1D systems play a key role for elucidating the physical properties of solids because many features of the electronic states can be discussed rigorously. It has been also confirmed that the ground state and lowlying excitations can be described within the framework of the Tomonaga-Luttinger-liquid (TLL) theory [12] even in the presence of both disorder and electron-electron interaction [10, 11]. Therefore, we are allowed to measure the effect of disorder as a modification of the TLL states. The properties of TLL are characterized by a few quantities; most notably, the charge exponent $K_{\rho}$ determines the long-range behavior of various correlation functions in the 1D metallic state. In this regard, a point to ponder is that the all eigenstates of a disordered $1 \mathrm{D}$ system are exponentially localized in the asymptotic sense [13].
In this Letter, we study a 1D Hubbard model in the presence of disorder, namely 1D Anderson-Hubbard (AH) model. Using the density-matrix renormalization group (DMRG) technique [14], the system-size dependent density-density correlation functions are calculated for various on-site Coulomb interactions and disorder strengths. We then propose a finite-size scaling method of the correlation functions for obtaining the modified TLL charge exponent and localization length of the electrons. In consequence, a quadratic decay of the localization length with the inverse of disorder strength is confirmed for any interaction and band filling. We also find a drastic reduction of the charge exponent with disorder except around quarter filling and a strong suppression of the localization length with the on-site Coulomb interaction near half filling.

The Hamiltonian of the 1D AH model is written as

$H=-t \sum_{i=1, \sigma}^{L-1}\left(c_{i \sigma}^{\dagger} c_{i+1 \sigma}+H . c.\right)+U \sum_{i=1}^{L} n_{i \uparrow} n_{i \downarrow}+\Delta \sum_{i=1, \sigma}^{L} \varepsilon_{i} n_{i \sigma}$

where $c_{i \sigma}$ is annihilation operator of an electron with spin $\sigma(=\uparrow$ or $\downarrow)$ at site $i, L$ is the system length, and $n_{i \sigma}=c_{i \sigma}^{\dagger} c_{i \sigma}$ is number operator. The nearest-neighbor hopping integral $t$ and on-site Coulomb interaction $U$ are assumed to be constant over the system. The random on-site potential $\varepsilon_{i}(i=1, \cdots, L)$ is defined by a box probability distribution $\mathcal{P}\left(\varepsilon_{i}\right)=\theta\left(1 / 2-\left|\varepsilon_{i}\right|\right)$ with the step function $\theta(x)$ and the disorder strength is controlled by $\Delta$. The band filling is $n=N / L$ where $N$ is the total number of electrons. We set $t=1$ as energy unit hereafter.

We consider the long-range behavior of the densitydensity correlation in the presence of disorder. We focus on the case of $U \geq 0$ and $0<\Delta \lesssim U / 2$, where the sys- 
tem is in the localized phase. Thus, the density-density correlation functions may be defined like [15, 16]

$$
C(r)=\left.e^{-\frac{\pi^{2} r}{6 \xi}} C(r)\right|_{\Delta=0}
$$

with the asymptotic behavior in the absence of disorder

$$
\left.C(r)\right|_{\Delta=0}=-\frac{K_{\rho}}{(\pi r)^{2}}+\frac{A \cos \left(2 k_{\mathrm{F}} r\right)}{r^{1+K_{\rho}}} \ln ^{-3 / 2}(r)+\cdots,
$$

where $\xi$ corresponds to the localization length of the electrons. Calculating the Fourier transformation of Eq.(2),

$$
C\left(q_{1}\right)=\sum_{r=1}^{L} C(r) e^{i q_{1} r},
$$

with $q_{1}=2 \pi / L$, we obtain

$$
C\left(q_{1}\right)=-\frac{K_{\rho}^{*}}{2 \pi^{2}} \frac{e^{-\frac{\pi^{2} L}{6 \xi}}-1}{e^{\frac{\pi^{2}}{6 \xi}}-1} q_{1}^{2}
$$

for $q_{1} \ll 1(L \gg 1)$. The parameter $K_{\rho}^{*}$ is interpreted as a modified TLL charge exponent due to the disorder. In the limit of weak disorder, $K_{\rho}^{*}$ is equivalent to $K_{\rho}$ and $\xi$ tends to be infinite. By the DMRG method [17], we calculate $C\left(q_{1}\right)$ on random sampling 300 (500) realizations of $\mathcal{P}\left(\varepsilon_{i}\right)$ for $L=128,112,96,80,64,48$, (32, and 16$)$; then, we take an average of the results for each system size in order to obtain physically meaningful values of $C\left(q_{1}\right)$ [The averaged value is denoted as $\bar{C}\left(q_{1}\right)$ ]. For accurate calculation, the open-boundary conditions (OBC) are applied and we keep up to $m=2400$ density-matrix eigenstates in the renormalization procedure. Note that special attention should be paid to the convergence of the calculation because the DMRG wave function is apt to get trapped in a 'false' ground state for disordered system. Thus, $K_{\rho}^{*}$ and $\xi$ will be estimated by fitting our numerical data of $\bar{C}\left(q_{1}\right)$ with Eq.(55).

For instance, we demonstrate the fitting of $\bar{C}\left(q_{1}\right)$ at $U=10$ and $n=1 / 2$. Figure1(a) shows the averaged values of $C\left(q_{1}\right)$ as a function of $1 /(L-\delta)$ for various disorder strengths $\Delta$. Since a certain quantity of the correlations is missing at system edges due to the OBC, $L$ should be replaced with $L-\delta(\delta>0)$ for an excellent finite-sizescaling analysis. The correction factor $\delta$, which would be of the order of the lattice spacing, is determined to reproduce the relation $K_{\rho}^{*}=K_{\rho}$ in the absence of disorder; as expected, we obtain a quasi-infinite localization length $\left(1 /|\xi| \lesssim 1.0 \times 10^{-5}\right)$ with $\delta \approx 0.8-1.2$ at $\Delta=0$ for all fillings and interaction strengths considered in this study. In this way, the DMRG results of $\bar{C}\left(q_{1}\right)$ are well fitted by Eq. (5D) even for finite $\Delta$ values, as shown in Fig.1(a). The estimated values of $K_{\rho}^{*}$ and $\xi$ are plotted as a function of $\Delta$ in Fig. 1 (b) and (c), respectively. The exponent $K_{\rho}^{*}$ is reduced with increasing $\Delta$ because the forward scattering processes are enhanced by the disorder [16]. However,
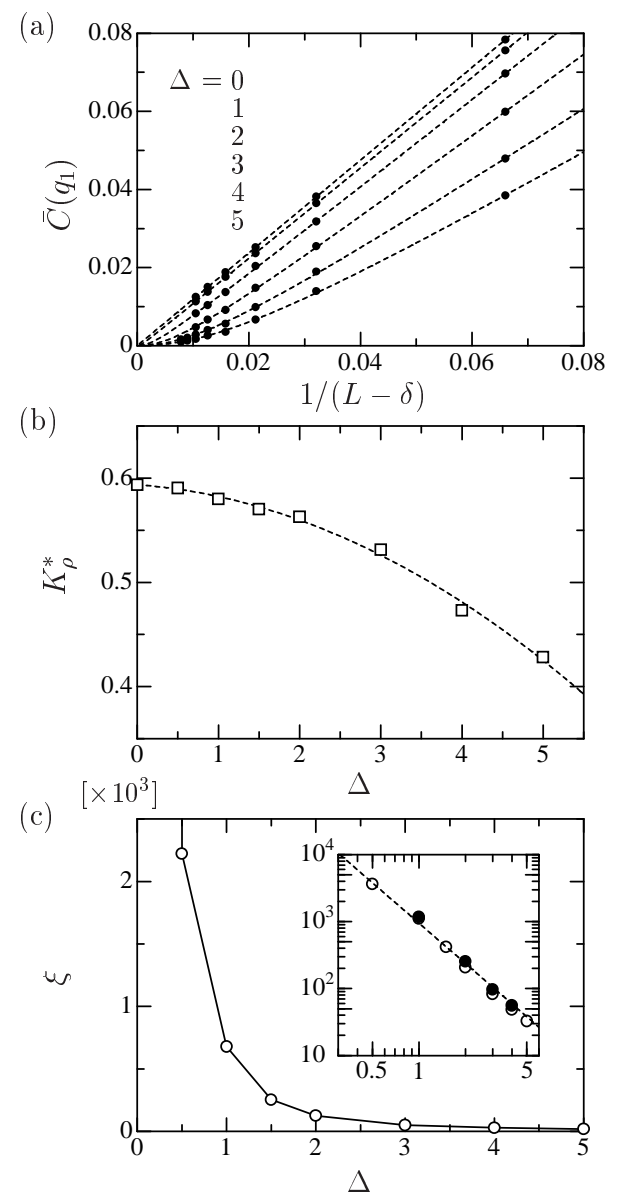

FIG. 1: (a) Finite-size-scaling analyses of the charge correlation function $\bar{C}\left(q_{1}\right)$ for various disorder strengths $\Delta$ at $U=10$. The dotted lines are the fitting with Eq. (5). (b) Fitting results of the TLL charge exponent $K_{\rho}^{*}$ as a function of $\Delta$. The dashed line is a second-order polynomial in $\Delta$. (c) Fitting results of the localization length $\xi$ as a function of $\Delta$. Insets: Log-log plot of $\xi$ with $\Delta$ (open circles). The values of $\xi$ estimated from the Drude weight are also plotted as solid circles.

the shortening of the localization length $\xi$ on $\Delta$ seems to be much more drastic. We find that $\xi$ decays as a power law with $\Delta$, i.e., $\xi=\xi_{0} \Delta^{-\alpha}$, if $\Delta \lesssim U / 2$. At $n=1 / 2$, we estimate $\xi_{0} \approx 950$ and $\alpha \approx 2$ [see the inset of Fig. [1(c)].

In order to check the accuracy of the above analysis, we also estimate the localization length from an exponential decay of the Drude weight $D(L)$ with the system length $L$ [18, 19] and perform a cross-check. The Drude weight is calculated on random sampling 100 realizations of $\mathcal{P}\left(\varepsilon_{i}\right)$ for $64,48,32$, and 16 using a recently proposed method 20]. The periodic boundary conditions are applied for this calculation. By fitting of the averaged results of $D(L)[=\bar{D}(L)]$ with a formula

$$
\bar{D}(L)=\gamma \exp \left(-\frac{L}{\xi}\right), \quad \gamma>0,
$$

we can obtain $\xi$ without any difficulty. As seen in the in- 
(a)

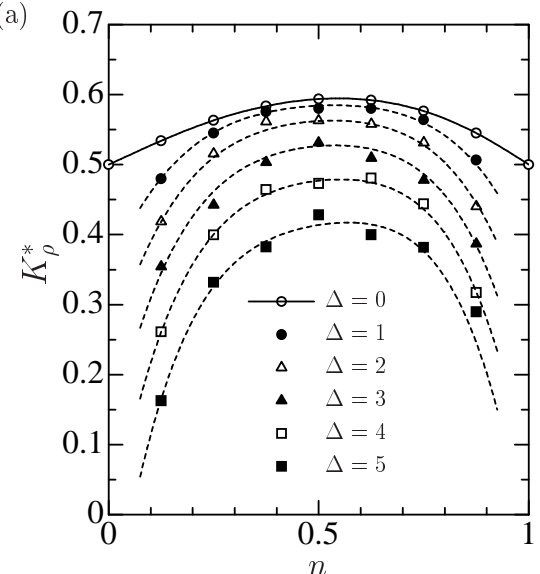

(b)

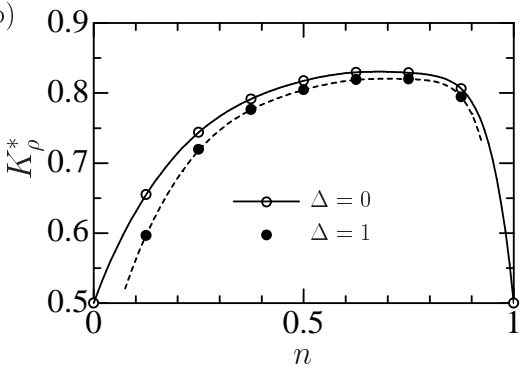

(a)

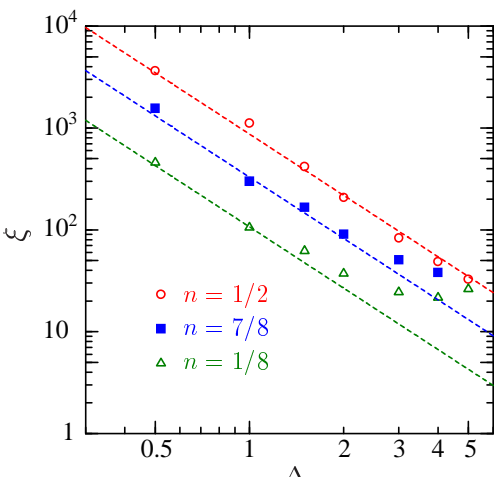

(b)

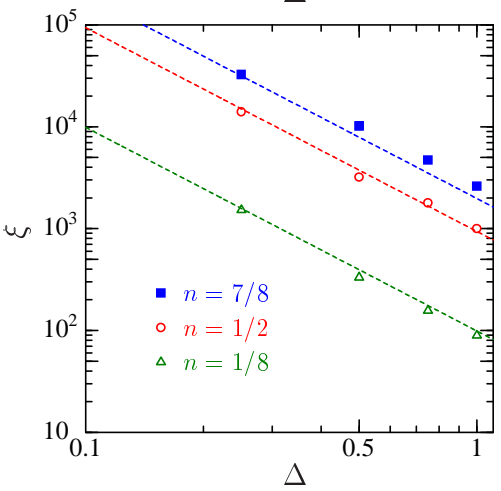

FIG. 2: Modified charge exponent $K_{\rho}^{*}$ as a function of the band filling $n$ for various values of the disorder strength $\Delta$ at (a) $U=10$ and (b) $U=2$. The dashed lines are guides to the eye.

set of Fig. 1(c), there is an excellent agreement between the results from $\bar{D}(L)$ and $\bar{C}\left(q_{1}\right)$. Therefore, we can confirm the validity of our method.

Let us now see how the charge exponent is modified by the disorder. Figure 2 shows the values of $K_{\rho}^{*}$ obtained by the above fitting procedure as a function of the band filling $n$ for various disorder strengths $\Delta$ at (a) strong $(U=10)$ and (b) weak $(U=2)$ interaction strengths. In the case of $U=10$, we see that the charge exponent is most severely affected by the disorder near $n=0$; namely, $K_{\rho}^{*}$ is drastically reduced by $\Delta$ since most of (or all) the electrons are easily trapped at sites having low on-site potential. Also near $n=1, K_{\rho}^{*}$ decreases rapidly with increasing $\Delta$. This can be explained in terms of the formation of Mott plateaus assisted by the disorder 21]. In other words, the system will be essentially in the Mott insulating state for tiny doping since the holes are strongly localized. On the other hand, the effect of disorder appears to be relatively weak around quarter filling because significant "untrapped" particles still remain and they could transfer. Consequently, the charge exponent is hardly affected for small $\Delta(\lesssim 1)$ at $n \approx 0.3-0.7$. It is consistent with a rather unstable Mott plateau far away from half filing 21]. We then turn to the case of $U=2$. Qualitatively the same behavior of $K_{\rho}^{*}$ with $\Delta$ is observed as long as the band filling is far away
FIG. 3: The localization length $\xi$ as a function of the disorder strength $\Delta$ for (a) $U=10$ and (b) $U=2$. The dashed lines are the fitting function $\xi=\frac{\xi_{0}}{\Delta^{2}}$ for small $\Delta$.

from half filing and, however, the effect of $\Delta$ near half filling seems to be weaker in comparison with the case of $U=10$. It would be concerned with the fact that the formation of the Mott plateau is harder for smaller $U$.

Of particular interest is the evolution of the localization length $\xi$ with increasing the disorder strength $\Delta$. Figure 3 shows the fitting values of $\xi$ as a function of $\Delta$ for several kinds of the band filling $n$ at (a) $U=10$ and (b) $U=2$. We find that the localization length always decay quadratically with the inverse of the disorder strength, i.e.,

$$
\xi=\frac{\xi_{0}}{\Delta^{2}}
$$

when the disorder is weak. The same relation has been already proposed for spinless fermions case $(U=$ () 22, 23], which is the same model as what proposed by Anderson [1]. Therefore, the universality of Eq.(77) is confirmed in a weakly disordered system independently of the on-site Coulomb interaction and band filling. We note that the localization length begins to deviate from Eq.(77) at relatively small disorder $\Delta \approx 1.5-2$ near $n=0,1$ for $U=10$. It is because a part of the sites is doubly occupied under the strong disorder and the density-density correlation no longer obeys Eq.(2).

A qualitative determination of the decay ratio $\xi_{0}$ in Eq.(77) is another challenging problem. So far, it has been 


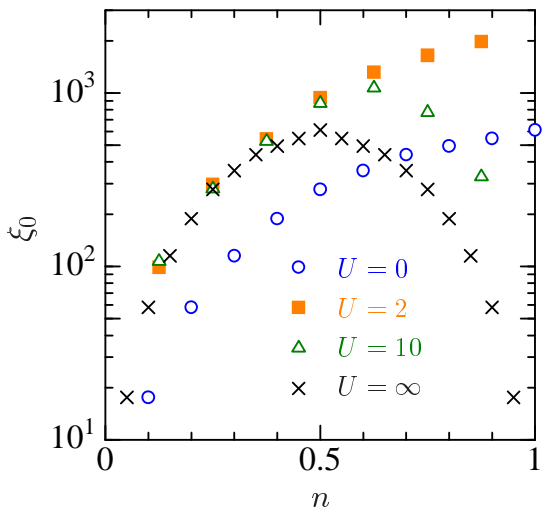

FIG. 4: Fitting values of the decay ratio $\xi_{0}$ for various interaction strengths.

studied perturbatively 22] and numerically [19, 24, 25] only for spinless fermions. Figure 4 shows the fitting values of $\xi_{0}$ as a function of the band filling $n$ at $U=0,2$, 10 , and $\infty$. When the interaction strength is varied from $U=0$ to $2, \xi_{0}$ increases for all fillings. It seems to be consistent with a prediction that the persistent currents are enhanced by the repulsive interactions 26] and, however, $\xi_{0}$ decreases with further increasing $U$. Especially near $n=1$, the reduction of $\xi_{0}$ with $U$ is quite rapid in connection to the stabilization of the Mott plateaus. On the other hand, surprisingly, $\xi_{0}$ is almost unchanged at $n \lesssim 0.5$ for $U \gtrsim 2$. Consequently, $\xi_{0}$ becomes symmetric about $n=1 / 2$ at large $U$, which reflects the particle-hole symmetry in the limit of $U \rightarrow \infty$.

In summary, we consider the 1D Anderson-Hubbard model using the DMRG technique. A finite-size scaling method of the density-density correlation functions for obtaining the modified TLL charge exponent and localization length is demonstrated. As results, we find a quadratic decay of the localization length with the inverse of disorder strength for any interaction and band filling, a drastic reduction of the TLL charge exponent with disorder except around quarter filling and, a strong suppression of the localization length with the on-site Coulomb interaction near half filling.

Lastly, we make a short remark regarding an explanation of the pseudogap-like behavior and very small TLL exponent $K_{\rho} \approx 0.23$ [27] observed in the TMTSF salts, which is quarter-filled system with dimerization. Regarding the dimer (TMTSF) $)_{2}$ as a site, it may be reduced to a half-filled system. Thus, we will expect a strong reduction of the TLL exponent even by tiny disorder. Moreover, if the relation $A(E) \sim\left|E-E_{\mathrm{F}}\right|^{\alpha}$ with
$\alpha=\left(K_{\rho}+K_{\rho}^{-1}-2\right) / 4$ is still valid in the presence of the disorder, the strong reduction of $K_{\rho}$ would be compatible with the pseudogap-like behvbior.

[1] P.W. Anderson, Phys. Rev. 109, 1492 (1958).

[2] For a review, B. Kramer and A. MacKinnon, Rep. Prog. Phys. 56, 1469 (1993).

[3] For a review, M. Lewenstein, A. Sanpera, V. Ahufinger, B. Damski, A. Sen, and U. Sen, Adv. Phys. 56, 243 (2007).

[4] A.L. Efros and B.I. Shklovskii, J. Phys. C 8, L49 (1975).

[5] S. Chiesa, P.. Chakraborty, W.E. Pickett, and R.T. Scalettar, arXiv:0804.4463v1.

[6] Y. Song, S. Bulut, R. Wortis, W.A. Atkinson, arXiv:0808.3356 1 .

[7] H. Shinaoka and M. Imada, Phys. Rev. Lett. 102, 016404 (2009).

[8] V. Vescoli1, F. Zwick, W. Henderson, L. Degiorgi, M. Grioni, G. Gruner, and L.K. Montgomery, Eur. Phys. J. B 13, 503 (2000).

[9] M. Sing, U. Schwingenschlögl, R. Claessen, M. Dressel, and C.S. Jacobsen, Phys. Rev. B 67, 125402 (2003).

[10] D. Loss, Phys. Rev. Lett. 69, 343 (1992).

[11] A.O. Gogolin and N.V. NV Prokof'ev, Phys. Rev. B 50, 4921 (1994).

[12] J. Sólyom, Adv. Phys. 28, 201 (1979).

[13] E. Abrahams, P.W. Anderson, D.C. Licciardello, and T.V. Ramakrishnan, Phys. Rev. Lett. 42, 673 (1979).

[14] S.R. White, Phys. Rev. Lett. 69, 2863 (1992); Phys. Rev. B 48, 10345 (1993).

[15] T. Saso, Y. Suzumura, and H. Fukuyama, Prog. Theor. Phys. Suppl. 84, 269 (1985).

[16] T. Giamarchi and H.J. Schulz, Phys. Rev. B 37, 325 (1988).

[17] S. Ejima, F. Gebhard, and S. Nishimoto, Europhys. Lett. 70, 492 (2005).

[18] C.L. Kane and M.P.A. Fisher, Phys. Rev. Lett. 68, 1220 (1992).

[19] P. Schmitteckert, T. Schulze, C. Schuster, P. Schwab, and U. Eckern, Phys. Rev. Lett. 80, 560 (1998).

[20] T. Shirakawa and E. Jeckelmann, in preparation.

[21] M. Okumura, S. Yamada, N. Taniguchi, and M. Machida, Phys. Rev. Lett. 101, 016407 (2008).

[22] D.J. Thouless, J. Phys. C 5. 77 (1972).

[23] D.C. Herbert and R. Jones, J. Phys. C 4, 1145 (1971).

[24] G. Czycholl, B. Kramer, and A. MacKinnon, Z. Phys. B 43, 5 (1981).

[25] M. Kappus and F.J. Wegner, Z. Phys. B 45, 15 (1981).

[26] T. Giamarchi and B.S. Shastry, Phys. Rev. B 51, 10915 (1995).

[27] M. Dressel, A. Schwartz, G. Grüner, and L. Degiorgi, Phys. Rev. Lett. 77, 398 (1996). 